\title{
Comparaison de l'évolution de l'indice de réfraction et de la viscosité au cours de la gélification lactique du lait
}

\author{
Marie-Hélène FAMELART et J.L. MAUBOIS \\ I.N.R.A., Laboratoire de Recherches de Technologie laitière \\ 65, rue de Saint-Brieuc, 35042 Rennes Cedex, France
}

\section{Résumé}

Des gélifications acides de lait par Streptococcus thermophilus (CNRZ 7 et CNRZ 385) sont suivies par des mesures de viscosité à l'aide d'un viscosimètre à cylindres coaxiaux, ainsi que par des mesures à l'aide d'un capteur réfractométrique. La viscosité et l'indice de réfraction (IR) sont fonction du $\mathrm{pH}$, et augmentent simultanément à $\mathrm{pH} 5,4$. A pH 5,0, la viscosité mesurée chute brusquement tandis que l'IR augmente toujours. Des corrélations significatives ont été obtenues entre la viscosité dans sa partie montante et l'indice de réfraction. Elles conduisent à penser que le réfractomètre utilisé pourrait constituer un capteur non destructif satisfaisant pour l'étude de la gélification du lait par des bactéries lactiques. Quelques hypothèses sont émises sur le phénomène capté par le réfractomètre.

Mots clés: Gélification acide - pH - Viscosité - Indice de réfraction - Streptococcus.

\section{Summary}

Comparison of refractive index and viscosity evolution during lactic gelification of milk.

A coaxial cylinders viscosimeter coupled with a refractometer were used to follow acid gelification of milk with Streptococcus thermophilus (CNRZ 7 and CNRZ 385). The viscosity and the refractive signal (IR) were functions of $\mathrm{pH}$ and increased simultaneously at $\mathrm{pH} 5.4$. At $\mathrm{pH} 5.0$, the viscosity decreased rapidly, while the IR still rised. Significant linear regressions between viscosity (restricted to the rising part of the viscosity time curves) and IR were obtained. These results show that the used refractometer could be a satisfactory non-destructive sensor for milk gelification with lactic acid bacteria. Some hypothesis are discussed concerning the phenomena followed by the refractometer.

Key words : Acid gelification - pH - Viscosity - Refractive index - Streptococcus.

\section{Introduction}

Si de nombreuses études ont été consacrées à la gélification enzymatique du lait (Thomasow et Voss, 1977; Green et Morant, 1981 ; Mc Mahon et 
al., 1984), peu l'ont été à la coagulation du lait par des bactéries lactiques. Dans la plupart des cas, cette gélification a été appréhendée par l'intermédiaire de simulations de la production graduelle d'acide par addition préalable de glucono-delta-lactone (Harwalkar et Kalab, 1980 ; HeertJe et al., 1985), ou bien d'autres systèmes acidogènes, comme l'anhydride propionique, les couples éthylbutyrate-estérase, tributyrine-lipase (Trop, 1984), par ajout d'acide chlorhydrique à froid $\left(0-4^{\circ} \mathrm{C}\right)$ suivi d'un chauffage progressif (HARWALKAR et KALAB, 1981 ; RoEFs, 1985). De même si quelques équipes travaillent sur la microstructure du yaourt (KALAB et al., 1976 et 1983 ; ModLer et al., 1983 ; SchellhaAss et Morris, 1985), peu s'intéressent à la cinétique de la formation du gel lors de la fermentation du lait par des levains lactiques.

Il est vrai que les méthodes d'appréhension des caractéristiques physiques des gels, reposant sur la mesure des forces s'opposant à un cisaillement contrôlé, conduisent à des résultats sujets à caution du fait de l'extrême fragilité mécanique des gels lactiques.

Les excellentes corrélations démontrées récemment par Korolczuk et al. (1986) entre l'évolution de la viscosité et de l'indice de réfraction lors de la gélification présure du lait nous ont amenés à penser que la formation des gels lactiques pouvait être suivie avec le même appareillage. Il était, en effet, vraisemblable que le changement d'état physique d'un lait soumis à l'acidification par le développement de bactéries lactiques devait conduire à une modification du signal optique émanant du réfractomètre qui, au contraire du viscosimètre à cylindres coaxiaux, ne détruit pas la structure du gel en cours de formation.

Pour vérifier cette hypothèse, nous avons étudié au cours de ce travail préliminaire, la cinétique de gélification du lait ensemencé avec deux souches de $S$. thermophilus, l'une à vitesse d'acidification lente, l'autre rapide. Un tel choix a été fait tant en raison de l'intérêt sensu stricto de $S$. thermophilus, une des 2 bactéries essentielles de la fabrication du yaourt, que dans le but d'appréhender d'éventuelles différences des caractéristiques de gel liées à la vitesse d'acidification.

\section{Matériel et méthodes}

\section{A. Lait utilisé}

Une poudre de lait « Medium-heat " «Elle-et-Vire » était reconstituée à $10 \mathrm{~g} / 100 \mathrm{ml}$ dans de l'eau distillée à $80^{\circ} \mathrm{C}$ puis refroidie et conservée une nuit à $4{ }^{\circ} \mathrm{C}$. Le $\mathrm{pH}$ était ajusté à 6,6 , et le lait maintenu dans un flacon 1 heure au bain d'eau à la température de l'essai avant l'ensemencement.

\section{B. Souches}

Les souches Streptococcus thermophilus CNRZ 7 et CNRZ 385 (collection de l'Institut National de la Recherche Agronomique) étaient conservées à l'état lyophilisé et remises en culture sur lait tournesolé autoclavé à $37^{\circ} \mathrm{C}$ (2 repi- 
quages à $2 \%$, puis un repiquage à $5 \%$ ). Un stock était alors constitué à partir de lait tournesolé ensemencé à $5 \%$, non incubé, congelé à $-25^{\circ} \mathrm{C}$. Les cultures étaient rapidement décongelées dans l'eau à $40^{\circ} \mathrm{C}$, incubées 10 heures à $37{ }^{\circ} \mathrm{C}$ et les précultures obtenues par repiquage à $5 \%$ sur lait autoclavé (10 heures).

\section{Appareillage utilisé}

L'équipement utilisé était celui précédemment décrit par KorolczuK et al. (1986). Il se composait d'un viscosimètre à cylindres coaxiaux (Rhéomat 30, CONTRAVES, Suisse) dont le cylindre extérieur, à double paroi, sert à la thermostatation du lait ensemencé. Au fond de ce cylindre extérieur, et dans la partie non agitée du lait, est fixé le réfractomètre SOPELEM (France). Le gradient de vitesse du viscosimètre était de $1267 \mathrm{~s}^{-1}$ pour toutes nos expérimentations.

\section{Cultures}

$120 \mathrm{ml}$ de lait étaient ensemencés à l'aide de $6 \mathrm{ml}$ de préculture dans le flacon placé dans le bain d'eau, agités puis rapidement transvasés dans le viscosimètre où la culture avait lieu. Cinq cultures à $42^{\circ} \mathrm{C}$ et 2 cultures à $37{ }^{\circ} \mathrm{C}$ (températures optimales) ont été réalisées respectivement pour les souches 385 et 7 , connues comme ayant des vitesses d'acidification sur lait très différentes.

Une culture de contrôle (10) de la souche 385 réalisée selon le même mode opératoire a été suivie plus complètement : des dosages d'acide lactique réalisés par HPLC sur colonne Aminex $\mathrm{A} 6$ avec $\mathrm{H}_{2} \mathrm{SO}_{4} 10^{-2} \mathrm{~mole} / \mathrm{l}$, à un débit de $1 \mathrm{ml} / \mathrm{mn}$ et à une longueur d'onde $214 \mathrm{~nm}$ (BRule, 1985), des numérations microbiennes sur milieu M17 gélosé, ainsi que des observations microscopiques ont été effectués.

\section{E. Acquisition des données}

Les 2 capteurs étaient connectés à un microordinateur de type Apple II Plus par l'intermédiaire de 2 voltmètres Philips (PM 2519 et PM 2534) et d'un bus IEEE. Le logiciel de saisie était celui décrit par Korolczuk et al. (1985). Les moyennes arithmétiques de 22 mesures de chaque capteur (viscosité en $\mathrm{mPas}$ et indice de réfraction en volts), ainsi que les temps étaient enregistrés sur disque souple. Une sonde $\mathrm{pH}$ placée dans la partie supérieure du viscosimètre, ainsi qu'un pHmètre (CRISON, 501 précision 0,01 unité $\mathrm{pH}$ ) relié à un enregistreur permettait de suivre l'acidification.

Le lissage des données provenant du Rhéomat 30 était effectué en substituant 3 fois successivement la mesure au temps $t$ par la moyenne arithmétique des mesures aux temps $\mathrm{t}-\Delta \mathrm{t}$, $\mathrm{t}$ et $\mathrm{t}+\Delta \mathrm{t}, \Delta \mathrm{t}$ étant l'intervalle de temps entre 2 mesures enregistrées, soit 10 secondes; puis les moyennes arithmétiques de 9 mesures de chaque capteur étaient reprises par un logiciel de tracé de courbes. La précision (coefficient de variation) des mesures était respectivement de $3 \%$ et $0,1 \%$ de la valeur initiale, pour les capteurs viscosimétrique et réfractométrique. 


\section{Résultats}

\section{A. Acidification et croissance}

Sur l'ensemble des cultures effectuées (fig. 1), le $\mathrm{pH}$ initial de 6,4 (résultant de l'addition au lait reconstitué de 5 p.100 de levain à pH 4,3) chutait à environ 6,1 au cours de la première heure de culture, et se stabilisait à cette valeur pendant une heure pour la souche 385 et deux heures pour la souche 7. Dans le cas de la culture 10 (souche 385 ), cette chute de $\mathrm{pH}$ s'accompagnait d'une augmentation de la concentration en acide lactique de 0,22 à $0,58 \mathrm{~g} / 1$, tandis que la population dénombrable passait de $2,9.10^{7}$ à $14,7.10^{7}$ colonies par $\mathrm{ml}$. Un allongement des chainettes de streptocoques était observé au microscope. Un lait témoin non ensemencé auquel est ajouté soit une solution d' $\mathrm{HCl}$ de $\mathrm{pH}$ voisin de 4,3 soit un lait acidifié par $\mathrm{HCl}$ à $\mathrm{pH} 4,30$ ne présente aucune chute de $\mathrm{pH}$ durant l'heure suivant l'ajout.

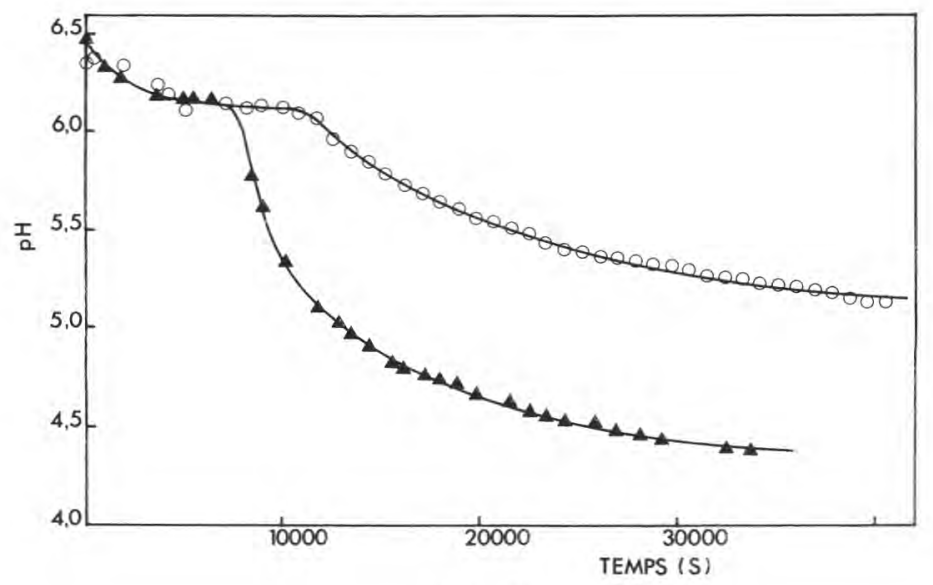

Fig. 1

Evolution du $\mathrm{pH}$ en fonction du temps pour 2 souches de $\mathrm{S}$. thermophilus : culture 2 (souche $385-\mathbf{\Delta}-$ ) et culture 30 (souche $7-0-$ ). Les résultats sont semblables pour les autres cultures de ces souches.

Evolution of $\mathrm{pH}$ as a function of time for 2 cultures of $\mathrm{S}$. thermophilus : culture 2 (strain $385-\mathbf{-}$ ) ) and culture 30 (strain $7-\mathrm{O}_{-}$). Results are similar for the other cultures of these strains.

Au-delà de la valeur 6,1 , le $\mathrm{pH}$ chutait rapidement surtout dans le cas de la souche 385 , et la population microbienne croissait exponentiellement. Dans le cas de la culture 10, la phase stationnaire était atteinte en moins de 3 heures, à $\mathrm{pH} 5,4$. Le nombre de streptocoques thermophiles était alors d'environ $1,4.10^{9}$ colonies par $\mathrm{ml}$ et n'augmentait plus, tandis que le $\mathrm{pH}$ atteignait à 8 heures de culture la valeur 4,4 pour la souche 385 . Dans le cas 
de la souche 7 , le $\mathrm{pH}$ avait atteint 5,4 en 8 heures, et nous n'avons pu atteindre des valeurs de $\mathrm{pH}$ de 4,4 , à cause de l'évaporation du lait devenant trop importante au-delà de 10 heures de culture.

\section{B. Viscosité et IR en fonction du pH}

Trois phases se dégageaient des courbes de gélification (fig. 2 et 3 pour chaque souche).

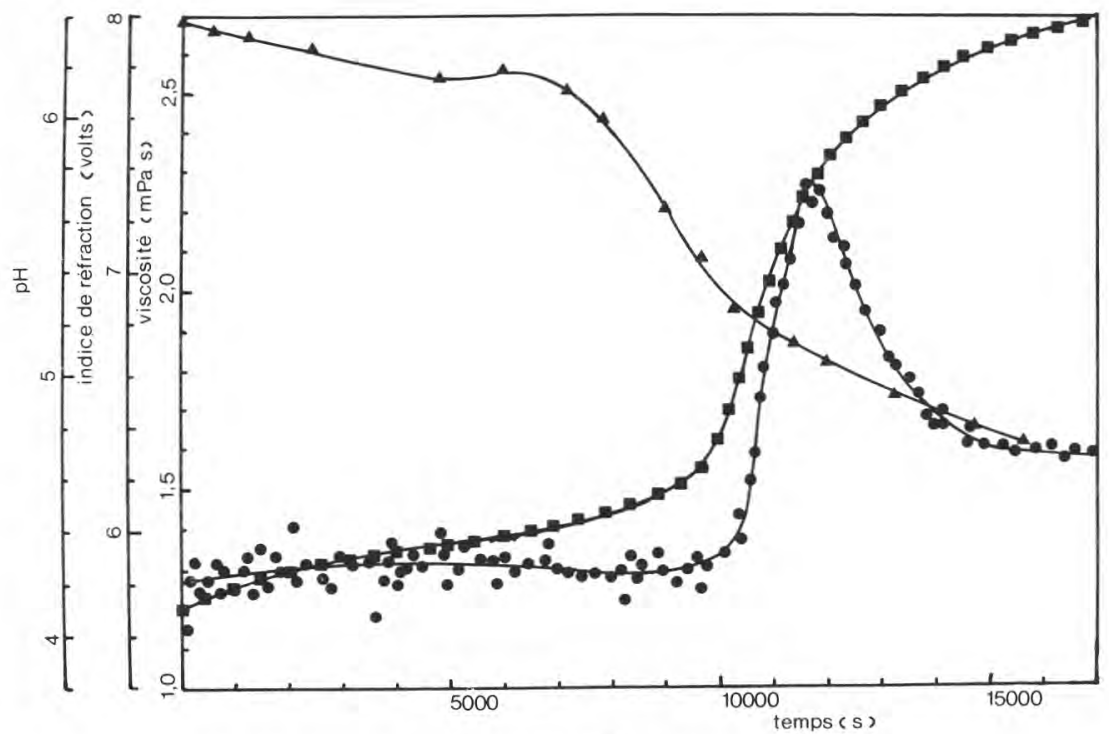

Fig. 2

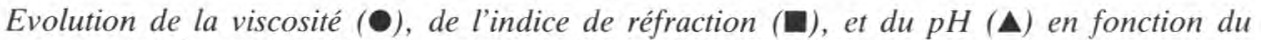
temps pour la souche 385 (culture 18). 1 point sur 2 est représenté.

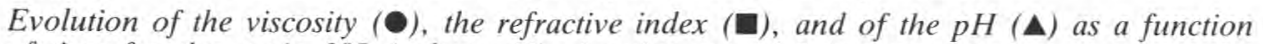
of time for the strain 385 (culture 18). 1 point out of 2 is represented.

- Pour les valeurs de pH allant de 6,4 à 5,4, la viscosité et l'IR augmentaient peu ou pas. Dans le cas de la souche 385 , la viscosité demeurait constante, tandis que l'IR augmentait de 0,5 à 0,8 volts. Pour la souche 7 , viscosité et IR augmentaient simultanément, avec un épaulement des 2 signaux à $\mathrm{pH}$ 6,0-6,2 (augmentation de 0,5 à 1 volt pour IR et de $0,2 \mathrm{mPa}$.s pour la viscosité). Cette augmentation initiale des 2 signaux lors des cultures de la souche 7 semblait être liée à l'acidification initiale du lait (abaissement de $\mathrm{pH}$ 6,4 à 6,1 ) tandis que ce phénomène n'était pas observé dans le cas de la souche 385 .

- Pour les valeurs de $\mathrm{pH}$ allant de 5,4 à 5,0 , la viscosité et l'IR augmentaient brusquement et simultanément. Le lait commençait alors à gélifier et une floculation était visible au microscope optique et à l'œil. 


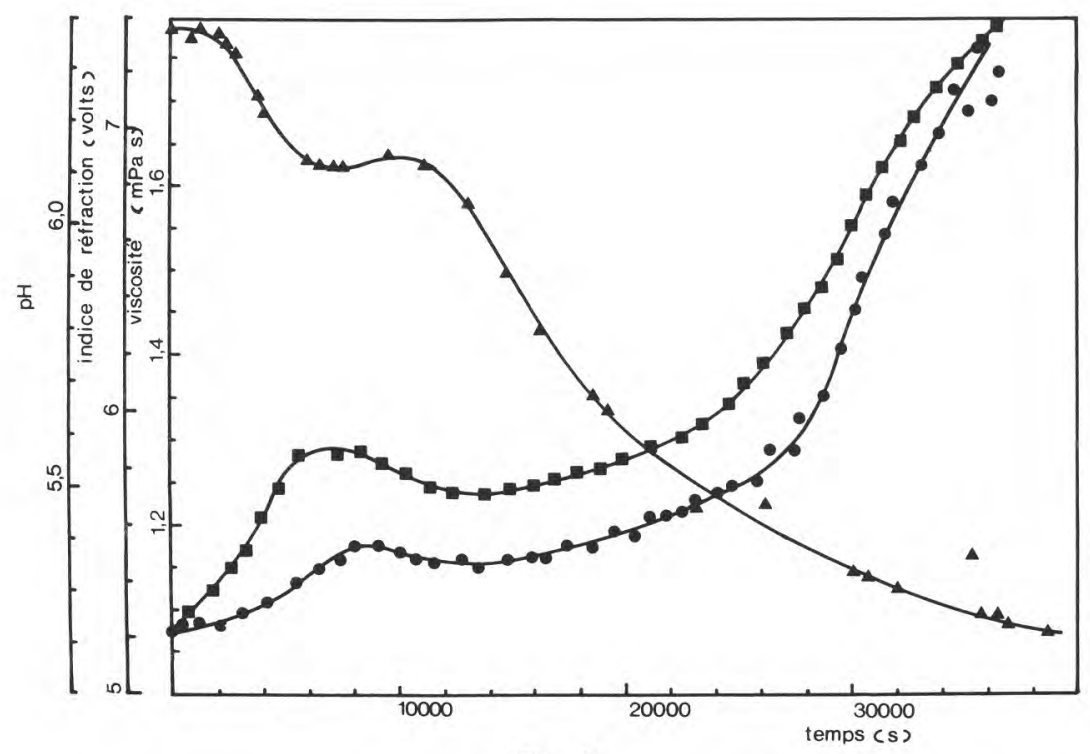

Fig. 3

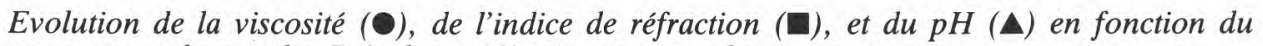
temps pour la souche 7 (culture 29). 1 point sur 8 est représenté.

Evolution of the viscosity (-), the refractive index (), and of the $p H(\mathbf{\Delta})$ as a function of time for the strain 7 (culture 29). 1 point out of 8 is represented.

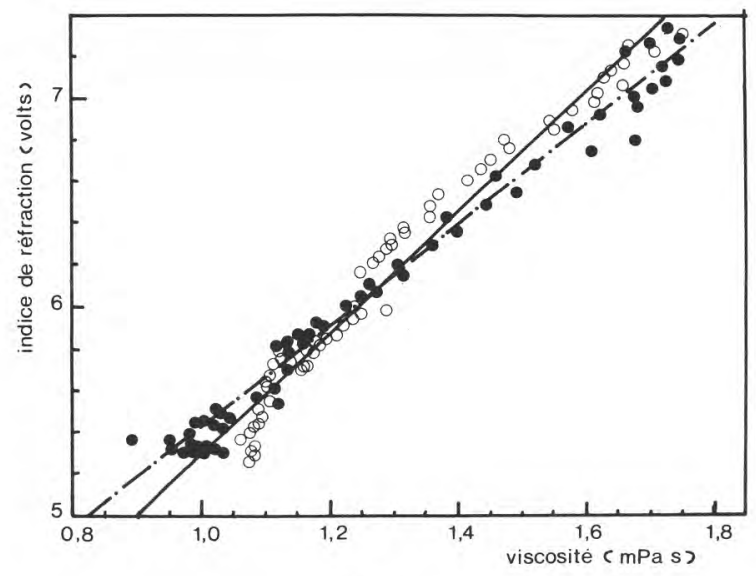

Fig. 4

Corrélations entre l'indice de réfraction et la viscosité pour les cultures de la souche 7 ( $O$ culture 29, - culture 30). Les traits continus représentent les droites de régression.

Correlations between the refractive index and the viscosity for the cultures of strain 7 (O culture 29, - culture 30). Lines are for the linear model. 
- Au-delà de $\mathrm{pH} 5,0$, tandis que l'IR augmentait toujours de façon importante, la viscosité chutait brusquement. Le gel formé était détruit par le cisaillement qui lui était imposé (gradient de vitesse $1267 \mathrm{~s}^{-1}$ ). Cette chute n'était pas visible dans le cas de la souche 7 qui acidifie plus lentement. Les viscosités maximales, ainsi que finales étaient variables pour les différentes cultures de la souche 385 (respectivement de 1,7 à $2,4 \mathrm{mPas}$ et de 1,4 à 1,9 $\mathrm{mPas}$ ), mais semblaient dépendre du niveau initial de la viscosité (corrélations significatives au seuil de 1 p. 100).

La déstructuration totale du gel par agitation violente dans la partie inférieure du cylindre externe de l'équipement de mesure ramenait l'IR à une valeur inférieure à celle observée sur le lait de départ.

Les données provenant du réfractomètre étaient bien moins dispersées que celles provenant du viscosimètre. Le rapport de l'amplitude maximale du signal sur l'écart type des mesures était d'environ 30 et 500, respectivement pour les capteurs rhéologique et optique.

\section{Relations entre viscosité et IR}

Les figures 4 et 5 indiquent les relations entre IR et viscosité, le tableau 1 fournit les valeurs des paramètres des droites de régression. Toutes les corrélations étaient significatives au seuil 1 pour 100 . L' ordonnée à l'origine était

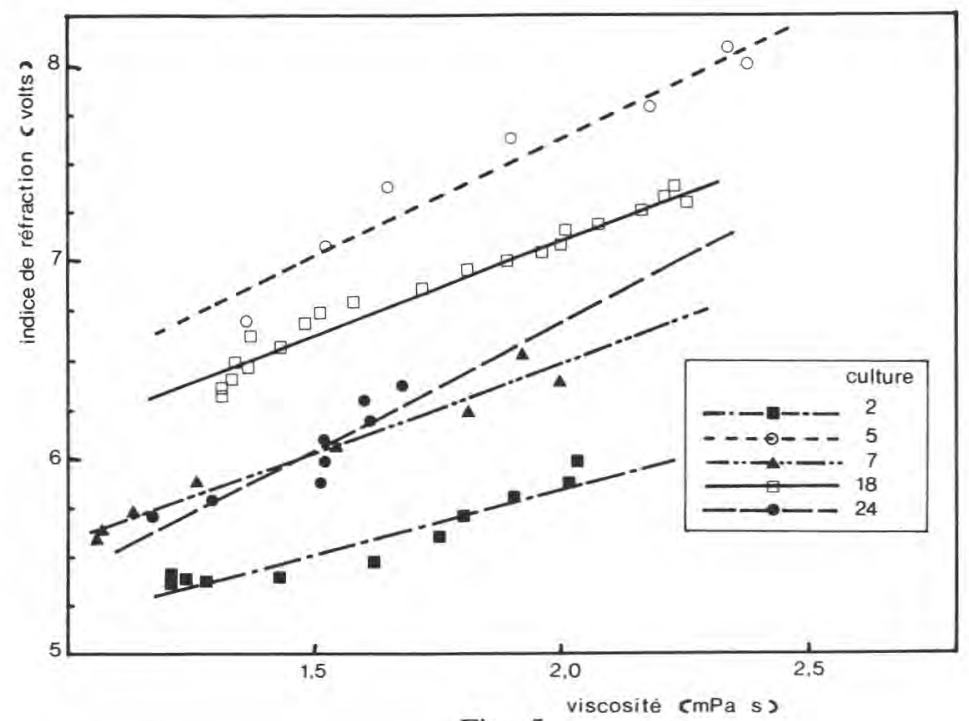

Fig. 5

Corrélations entre l'indice de réfraction et la viscosité pour les 5 cultures de la souche 385 (seule la partie montante de la courbe viscositéltemps est représentée). Les points représentent les points expérimentaux et les traits continus la droite de régression.

Correlations between the refractive index and the viscosity for the 5 cultures of strain 385 (only the rising part of viscosity/time curves is represented). Points are for experimental observations and lines are for the linear model. 
TABLEAU 1

Paramètres des droites de régression entre indice de réfraction et viscosité $[I R=f$ (viscosité) $]$. Pour la souche 385, les données comprennent uniquement la partie croissante des courbes viscositéltemps

Linear regression parameters between the refractive index and the viscosity $[I R=f$ (viscosity) $]$. For the strain 385 , the data concern only the rising part of viscosity/time curves

\begin{tabular}{|c|c|c|c|c|c|c|}
\hline Souche & Culture & $\mathrm{A}^{*}$ & $+1-* *$ & $\mathrm{~B}^{* * *}$ & $+1-$ & $\mathrm{r}^{* * * * *}$ \\
\hline 385 & $\begin{array}{r}2 \\
5 \\
7 \\
18 \\
24 \\
\end{array}$ & $\begin{array}{l}4,54 \\
5,22 \\
4,68 \\
5,22 \\
4,14 \\
\end{array}$ & $\begin{array}{l}0,134 \\
0,276 \\
0,141 \\
0,070 \\
0,499 \\
\end{array}$ & $\begin{array}{l}0,66 \\
1,21 \\
0,90 \\
0,94 \\
1,28 \\
\end{array}$ & $\begin{array}{l}0,218 \\
0,396 \\
0,180 \\
0,097 \\
0,614 \\
\end{array}$ & $\begin{array}{l}0,949 \\
0,973 \\
0,984 \\
0,984 \\
0,912 \\
\end{array}$ \\
\hline 7 & $\begin{array}{l}29 \\
30\end{array}$ & $\begin{array}{l}2,37 \\
3,01\end{array}$ & & $\begin{array}{l}2,92 \\
2,42\end{array}$ & & $\begin{array}{l}0,984 \\
0,989\end{array}$ \\
\hline 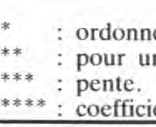 & $\begin{array}{l}\text { 'origine. } \\
\text { rvalle de } \\
\text { corrélati }\end{array}$ & e de & & & & \\
\hline
\end{tabular}

plus élevée pour les cultures de la souche 385 , mais les pentes étaient plus faibles que dans le cas de la souche 7. Pour une même augmentation de viscosité, l'augmentation de l'IR est au moins 2 fois plus forte dans le cas de la souche 7 que dans celui de la souche 385 . Les valeurs des pentes et des ordonnées à l'origine sont variables pour la souche 385 , mais compte tenu des intervalles de confiance dans l'estimation de ces paramètres (tabl. 1), l'ordonnée à l'origine et la pente sont respectivement proches de 5 et 1 .

\section{Discussion}

Les corrélations hautement significatives (fig. 4 et 5) existant entre les signaux de sortie du réfractomètre utilisé au cours du présent travail et les mesures de viscosité permettent d'affirmer que les deux capteurs mesurent le même phénomène, à savoir la formation d'un gel, sous l'effet de la bioconversion du lactose en acide lactique par $S$. thermophilus. Mais plus encore que pour la coagulation enzymatique du lait (KoRolczuk et al., 1986), la possibilité de suivre la formation des gels lactiques, très fragiles, à l'aide d'un capteur optique, non destructif, ouvre un champ d'investigations extrêmement vaste et diversifié : étude des caractéristiques physiques des gels, des souches bactériennes utilisées, de la composition et des traitements technologiques préalables appliqués au lait de fabrication. 
L'abaissement du pH entraîne une régression d'ionisation des fonctions acides des caséines. Il s'ensuit une diminution du potentiel de surface des micelles et un déplacement progressif du phosphate de calcium qui leur est lié vers la phase aqueuse (BRulé et LENOIR, 1987). II en résulte donc un accroissement de la teneur en sels minéraux de Ia phase soluble du lait.

L'abaissement du $\mathrm{pH}$ entraîne aussi une réduction progressive de l'hydratation et une déstructuration des micelles par rupture des ponts phosphocalciques liant les sous-unités entre elles. La taille moyenne des solutés protéiques diminue donc (Roefs et al., 1985) et amène donc, si on se réfère à l'équation proposée par DumANSKI et PuCHKovski (1929) à un accroissement de l'indice de réfraction. Par ailleurs, la concentration en lactose décroît et celle de l'acide augmente, tandis qu'une petite partie des caséines est solubilisée. C'est probablement à l'ensemble de ces phénomènes que doit être attribuée la croissance lente du signal réfractométrique observée entre $\mathrm{pH} 6,4$ et $\mathrm{pH} 5,4$ (fig. 2 et 3 ).

La croissance rapide du signal réfractométrique à $\mathrm{pH} 5,4$ traduit la formation d'un réseau tridimensionnel résultant de la formation de liaisons électrostatiques et hydrophobes entre polymères caséiques insolubilisés du fait de la neutralisation de la charge nette de surface et de la réduction maximale d'hydratation (BRULÉ et LENOIR, 1987). Le pH où débute la gélification est de 5,1 à $43^{\circ} \mathrm{C}$ pour un lait non chauffé tandis qu'il est de 5,5 pour un lait chauffé $\left(90^{\circ} \mathrm{C}-10 \mathrm{mn}\right.$ ) à $43^{\circ} \mathrm{C}$ (HeErtje et al., 1985). Les résultats des figures 2 et 3 permettent d'observer des $\mathrm{pH}$ de gélification de 5,4 .

Pas plus que pour les observations réalisées lors de la coagulation du lait sous l'effet de la présure, nous ne sommes en mesure de formuler une hypothèse satisfaisante pour expliquer la variation rapide du signal réfractométrique lors de la formation du gel. Y a-t-il véritablement variation de l'indice de réfraction - la désagrégation du gel formé par agitation ramenant la valeur du signal réfractométrique à une valeur inférieure ou égale à celle du lait de départ ne va pas dans le sens d'une telle interprétation - ou, ne serait-ce pas plutôt un phénomène de diffraction de la lumière qui serait détecté par le capteur réfractométrique utilisé ? Faute de données approfondies sur les relations existant entre les composantes du signal émis par le capteur et les caractéristiques optiques du produit testé, nous ne pouvons que constater le phénomène. Il doit également être noté, que toutes conditions étant égales, l'amplitude de variation du signal réfractométrique lors de la gélification lactique est au moins le double (plus de 2 volts contre 1 volt) de celle constatée lors de la gélification présure (Korolczuk et al., 1986). Si le phénomène détecté est bien lié à la diffraction de la lumière du rayon incident par le gel formé, un gel lactique serait donc deux fois plus diffractant que le gel présure et le capteur optique utilisé dans cette étude détecterait la différence de nature des mécanismes de coagulation existant entre action présure et action lactique, ce que ne fait pas le viscosimètre à cylindres coaxiaux.

Le capteur réfractométrique détecte également, si on s'en réfère aux figures 4 et 5 et au tableau 1, des différences de formation du gel liées à des différences de développement des 2 souches de bactéries lactiques. A partir des coefficients de régression contenus dans le tableau 1 , une viscosité de 
$2 \mathrm{mPas}$ correspond à une valeur moyenne de l'IR de 6,8 volts pour la souche 385 , à acidification rapide, et de 8 volts pour la souche 7 à acidification lente.

La comparaison des mesures viscosimétriques effectuées lors de cette étude avec celles de KorolczuK et al. (1986) montre que dans le cas de la gélification acide, l'accroissement de viscosité est plus faible et la chute après le maximum plus rapide et plus forte que dans le cas de la gélification présure. Il est à noter que la déstructuration complète des gels acides intervient toujours à $\mathrm{pH} 5,0$. Il est possible qu'à cette valeur de $\mathrm{pH}$ la taille des agrégats dépasse une valeur critique, compte tenu du cisaillement créé ; il s'ensuivrait alors une rupture des agrégats.

Le ralentissement de l'abaissement du $\mathrm{pH}$ constaté au niveau de la valeur 6,1 , observé pour toutes les cultures de façon plus ou moins marquée, se traduit pour la souche 7 par un ralentissement concomittant de l'accroissement de la viscosité et du signal réfractométrique. Un tel phénomène n'a pas été décrit, à notre connaissance, dans la littérature, et ne peut s'expliquer par le pouvoir tampon du lait, se situant plutôt aux environs de $\mathrm{pH} 5,2$. Cette observation doit être due au fait que, contrairement aux travaux de la littérature, nos cultures sont fortement agitées. Il peut s'agir soit d'un effet lié à l'homogénéisation des quantités d'acide produit, ou soit à un effet de l'aération du milieu entraînant une réorientation temporaire à ce niveau de $\mathrm{pH}$ des activités métaboliques des souches de $S$. thermophilus.

Reçu le 19 mars 1987. Accepté pour publication le $1^{\text {er }}$ juin 1987.

\section{Références bibliographiques}

Brulé G., 1985. Communication personnelle.

Brulé G., Lenolr J., 1987. La coagulation du lait. In : Le Fromage, ed.Eck A., Technique et Documentation, Lavoisier, Paris, 1-21.

Dumanski A., Puchkovski B.S., 1929. Kolloid-Ztg, 48, 338. Cité dans : Casein and its industrial application, Sutermeister E., Browne F.L. ed., 1939. Reinhold Publ. New York.

Green M.L., Morant S.V., 1981. Mechanism of aggregation of casein micelles in rennet-treated milk. J. Dairy Res., 48, 57-63.

Harwalkar V.R., Kalab M., 1980. Milk gel structure XI Electron microscopy of glucono- $\Delta$ lactone-induced skim milk gels. J. Texture Stud, 11, 35-49.

HaRwalkar V.R., Kalab M., 1981. Effect of acidulants and temperature on microstructure, firmness and susceptibility to syneresis of skim milk gels. Scanning Electron Microsc., 3, 503513.

Heertie I., Visser J., Smits P., 1985. Structure formation in acid milk gels. Food Microstruct., 4 , 267-277.

Kalab M., Allan-Wojtas P., Phipps-Todd B.E., 1983. Development of microstructure in Set-style non-fat yoghurt - A review. Food Microstruct., 2, 51-66.

Kalab M., Emmons D.B., SARGant A.G., 1976. Milk gel structure V. Microstructure of yoghurt as related to the heating of milk. Milchwissenschaft, 31 (7), 402-408.

Korolczuk J., Maubois J.-L., Loheac J., 1986. Suivi de la coagulation-présure du lait à l'aide d'un nouveau capteur réfractométrique. Lait, 66, 327-339.

Korolczuk J., Roignant M., Maubois J.-L., 1985. Computer data acquisition of sinusoidally varying stress and strain. J. Texture Stud., 16, 129-142. 
McMahon D.J., Brown R.J., Ernstrom C.A., 1984. Enzymic coagulation of milk casein micelles. J. Dairy Sci., 67, 745-748.

Modler H.W., Larmond M.E., Lin C.S., Froehlich D., Emmons D.B., 1983. Physical and sensory properties of yogurt stabilized with milk proteins. J. Dairy Sci., 66, 422-429.

RoEFS S.P.F.M., 1985. Structure of acid casein gels; a study of gels formed after acidification in the cold. Ph. D. thesis. Agricultural University, Wageningen.

Roefs S.P.F.M., Walstra P., Dalgleish D.G., Horne D.S., 1985. Preliminary note on the change in casein micelles caused by acidification. Neth. Milk Dairy J., 39, 119-122.

SChellhaAsS S.M., Morris H.A., 1985. Rheological and scanning electron microscopic examination of skim milk gels obtained by fermenting with ropy and non-ropy strains of lactic acid bacteria. Food Microstruct., 4, 279-287.

Thomasow J., Voss E., 1977. Methods for the determination of the firmness of milk coagulum. Bull. Fed. Int. Lait., (99), 1-12.

Trop M., 1984. Simulation of bacterial fermentation of milk and possible acylation of its proteins by acidogen hydrolysis. J. Dairy Sci., 67, 1381-1389. 